\title{
SEISMIC IMAGE SEGMENTATION
}

\author{
S. Wiseman \\ Center for Engineering Science Advanced Research (CESAR) \\ Computer Science and Mathematics Division \\ Oak Ridge National Laboratory \\ Oak Ridge, Tennessee 37831, USA
}

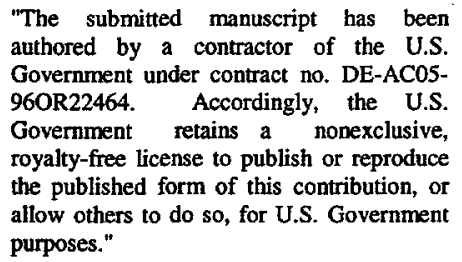

RECEIVED

MAR 031999

OSTI

Submitted: European Assoc. of Geoscientists and Engineers Conference, Helsinki, Finland, June 7-11, 1999

*Research supported in part by the DeepLook Consortium under agreement ERD-97-1506 with Lockheed Martin Energy Research Corporation and in part by the Engineering Research Program of the Office of Basic Energy Sciences and by the Office of Fossil Energy under contract DE-AC05-96OR22464 with Lockheed Martin Energy Research Corporation. 


\section{DISCLAIMER}

This report was prepared as an account of work sponsored by an agency of the United States Government. Neither the United States Government nor any agency thereof, nor any of their employees, make any warranty, express or implied, or assumes any legal liability or responsibility for the accuracy, completeness, or usefulness of any information, apparatus, product, or process disclosed, or represents that its use would not infringe privately owned rights. Reference herein to any specific commercial product, process, or service by trade name, trademark, manufacturer, or otherwise does not necessarily constitute or imply its endorsement, recommendation, or favoring by the United States Government or any agency thereof. The views and opinions of authors expressed herein do not necessarily state or reflect those of the United States Government or any agency thereof. 


\section{DISCLAIMER}

Portions of this document may be illegible in electronic image products. Images are produced from the best available original document. 


\title{
Seismic Image Segmentation
}

\author{
S. Wiseman \\ Center for Engineering Science Advanced Research (CESAR) \\ Computer Science and Mathematics Division \\ Oak Ridge National Laboratory \\ Oak Ridge, TN 37831, USA
}

\begin{abstract}
Image segmentation is a standard low-level task in computer vision. We demonstrate how the segmentation of seismic images can assist in geologic interpretation. An image segmentation technique is applied to images of the acoustic impedance. A salient feature of the resulting segments is that the hydrocarbon-bearing regions are characterized by a finer segmentation than the other regions. A modified apparent polarity attribute is introduced, and images of this attribute are segmented. The boundaries of the resulting segments pass through the minima and maxima of the acoustic impedance in a manner that allows us to segment the acoustic impedance image into layers of high and low acoustic impedance. Our methods can assist in segmentation of acoustic impedance images according to lithology or geologic facies.
\end{abstract}

The goal of geologic interpretation is to deduce from seismic data the existence and location of geological features such as sedimentary structures, stratigraphic boundaries, and rock lithologies[1]. Geological interpretation is often done by visual inspection of color-coded images of several acoustic attributes. Thus automatic image analysis techniques have the potential to assist in geological interpretation. Indeed, this direction was pursued by several authors and was argued for in [2]. Edge detection, which is similar to horizon picking, is an image analysis technique that can identify linear geological features such as faults, stratigraphic boundaries, etc. Image segmentation can potentially locate two-dimensional (2D) regions (segments) with relatively homogeneous properties such as the lithology or the stratigraphic orientation. The goal of image segmentation is to partition (segment) an image into connected regions of similar colors/gray levels or textures, with adjacent regions having significant dissimilarity. Image segmentation techniques (for a review see [3]) are widely applied in medical applications, industrial part inspection, land-cover analysis, and more. Some authors have attempted to segment seismic images according to stratigraphic patterns using textural features. $\mathrm{Ng}$, Kittler and Illingworth[4] succeeded in segmenting a salt dome from the sedimentary basin. Pitas and Kotropoulos[5] segmented seismic images using features such as reflection coefficient, horizon slope and horizon length. Simaan, Zhang and Love[6] combined texture image segmentation with a knowledge-based process. Aminzadeh and Chatterjee[7] demonstrated the utility of the closely related data clustering techniques in analyzing seismic sections.

In this work, we aim to segment seismic images so that the image segments correspond to terrain lithologies or geologic facies, which, in the oil field under study, correspond to different content ratios of sand and silt or clay. Two seismic attributes were found to be useful for this purpose: the acoustic impedance, $Z$, and a modified apparent polarity introduced in Sec. A. The image segmentation method that was used is sketched briefly in Sec. B. In Sec. C, we describe segmentation results for the acoustic impedance. In Sec. D, the segmentation results of the modified apparent polarity are shown and are used to segment the acoustic impedance image into alternating layers of high and low acoustic impedance.

$A$. Logarithmic apparent polarity The apparent polarity[8] can be defined as the product of the sign of the reflection coefficient $R$ with the reflection strength (or amplitude) $A_{a m p}$ :

$$
P=\operatorname{sign}(R) \cdot A_{a m p},
$$


where $A_{a m p}$ is the amplitude of the complex trace[8]. Recall that[1], assuming a normal interface between layer 1 lying on top of layer 2 , the reflection coefficient is $R \approx\left(Z_{2}-Z_{1}\right) /\left(Z_{1}+Z_{2}\right)$. Thus the reflection coefficient is positive (negative) when the acoustic impedance increases (decreases) with depth. Accordingly, a maximum, for instance, as a function of depth, of the acoustic impedance is accompanied by a change of $\operatorname{sign}(R)$ from +1 to -1 as depth increases. The apparent polarity is defined so that changes in sign $(R)$ are amplified when coinciding with high-amplitude events.

Usually, reflection strength (amplitude) images are displayed in color with a logarithmic color scale[8]. We also noticed that the probability distribution in our data of $A_{a m p}$ is similar to a log-normal distribution. Thus we defined a logarithmic apparent polarity as

$$
P_{L}=\operatorname{sign}(R) \cdot\left(\log A_{a m p}-\min \log A_{a m p}\right) .
$$

With this definition, $\operatorname{sign}(R)$ is weighted by the logarithm of the reflection strength. The subtraction of the minimum over the image of $\log A_{a m p}$ ensures that the weighting factor is positive so that $\operatorname{sign}\left(P_{L}\right)=\operatorname{sign}(R)$. The logarithmic apparent polarity image of Fig. 1(a) exhibits several advantageous properties: excellent contrast between positive and negative polarity regions and the existence of large segments that enhance lateral continuity.
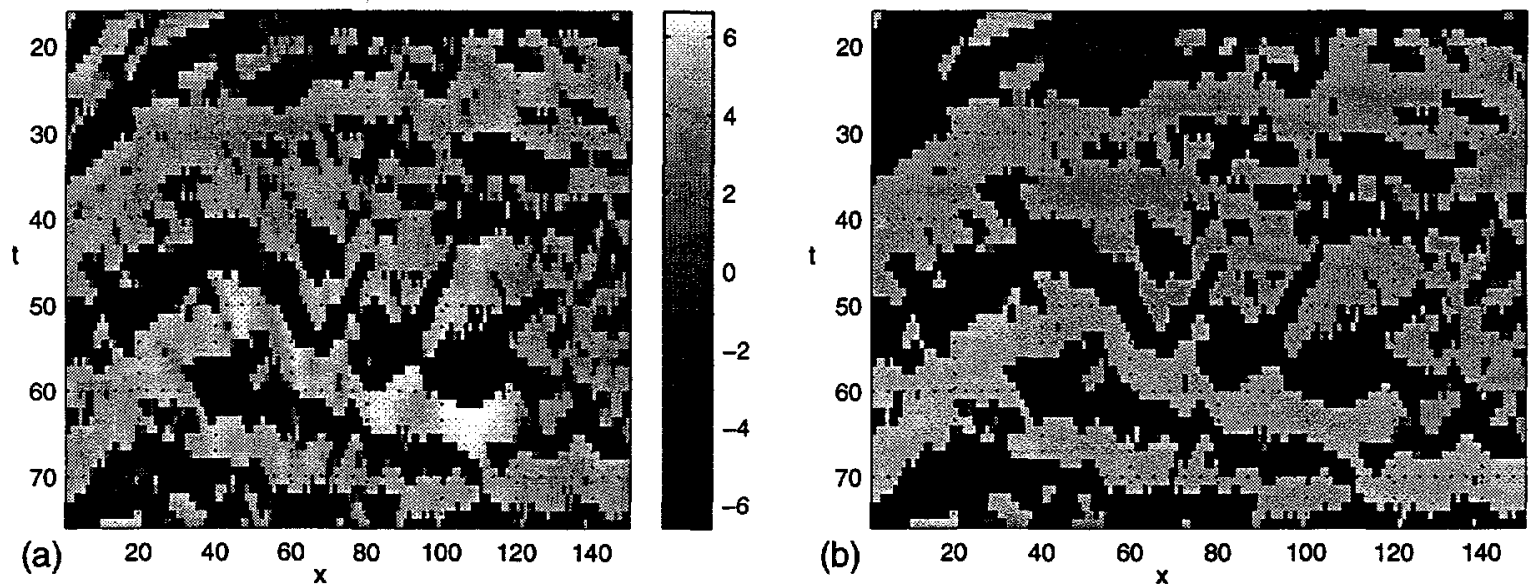

Figure 1: The original (a) and the segmented (b) logarithmic apparent polarity images.

$B$. The image segmentation method The image segmentation method is adapted from a data clustering method[9], both of which solve similar problems. Briefly, the segmentation method is outlined as follows. A classification $\mathcal{S}$ is defined by assigning to each pixel $i$ [the index $i$ corresponds to a pixel with some coordinates $\left.\left(x_{i}, y_{i}\right)\right]$ on the grid a label $s_{i}$, which may take integer values $s_{i}=1 \ldots q$. We define a cost function $H[\mathcal{S}]$ as

$$
H[\mathcal{S}]=-\sum_{\langle i, j\rangle}\left[J_{i j} \delta\left(s_{i}, s_{j}\right)\right]
$$

where the sum extends only over neighboring pixels $i$ and $j$ on the grid and $J_{i j}$ is a positive monotonically decreasing function of the distance $\left\|\vec{V}_{i}-\vec{V}_{j}\right\| . \quad V_{i}$ is the vector in feature space associated with pixel $i$. For example, when segmenting the acoustic impedance image $\vec{V}_{i}=Z_{i}$. The Kronecker delta function, $\delta\left(s_{i}, s_{j}\right)$, is one when $s_{i}=s_{j}$ and zero otherwise. Thus the closer two pixels are to each other (in feature space), the more they like to have the same label, since this lowers the cost. A probability distribution $P_{T}(\mathcal{S})$ is chosen that maximizes the unknown information[10] about $\mathcal{S}$ and yet has some fixed average cost $E$. The Lagrange multiplier $T$ associated with $E$ is a single control parameter of the method, which sets the resolution of the segmentation. Finally, the average [with respect to $P_{T}(\mathcal{S})$ ] label correlation, $\left\langle\delta\left(S_{i}, S_{j}\right)\right\rangle$, determines whether neighboring pixels $i$ and $j$ are in the same segment.

The data clustering method on which this image segmentation method is based is described in detail in [9]. The method was successfully applied to speech recognition, land-cover classification, and more[9]. The method is robust in the presence of noise, and its main advantage is in its generic 
applicability[9]. Further details of the image segmentation method and some specific details relevant to this work will be published elsewhere.

Our image segmentation method provides a roughly hierarchical sequence of image segmentations as a function of the "resolution parameter" $T$. For $T=0$, the whole image belongs to a single segment. As $T$ is increased, a hierarchy of segmentations, which are becoming finer and finer, is obtained. We chose a simple procedure for specifying a single final segmentation that seems to work reasonably. Starting at $T=0$, this procedure assigns each pixel to a segment at the lowest $T$ at which it no longer belongs to the largest segment. In a sense, this procedure is the coarsest nontrivial segmentation procedure. It is nontrivial because no pixel is allowed to belong to the largest segment of any temperature. Here we refer to a segmentation where all pixels belong to one segment as completely trivial. Because segments are assigned according to the lowest possible $T$ (but consistent with nontriviality), it is the coarsest.

$C$. Segmentation of the acoustic impedance The image of the acoustic impedance, $Z$, which is the product of the sound velocity with the density is shown in Fig. 2. In this Gulf of Mexico

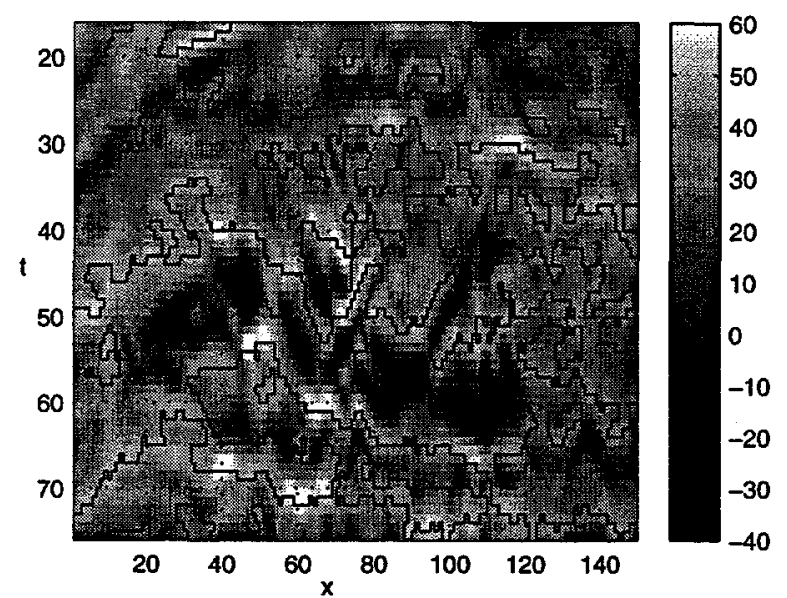

Figure 2: The acoustic impedance $Z$ of the $x-t$ image. Black lines mark the boundaries of the $P_{L}$ image segments (see Sec D). In all the images the horizontal inter-pixel spacing is 26.66 meters, while the vertical inter-pixel spacing is 4 milliseconds, which on average corresponded (according to the velocity model used in other analysis of the data) to $\sim 4$ meters. Axes are labeled in pixel units.
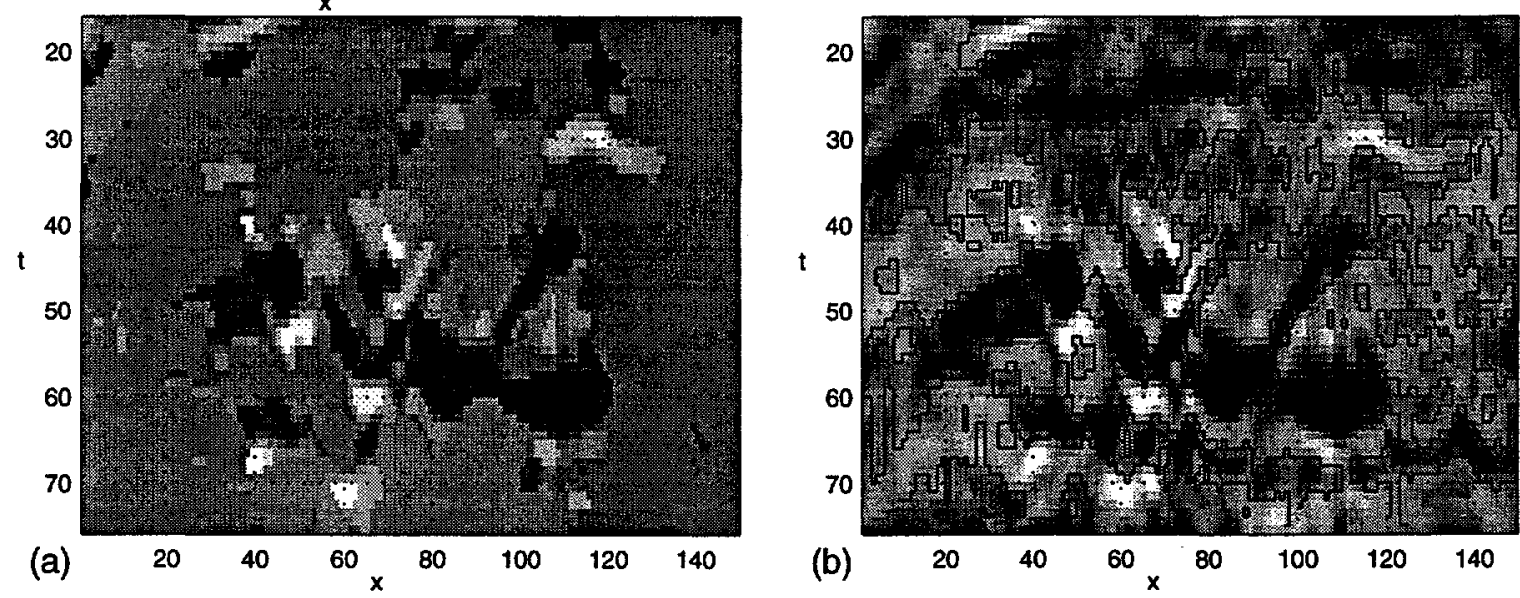

Figure 3: Acoustic impedance $Z$ with color scale of 2: (a) Segmented, with each segment colored according to the average value of $Z$ over all its pixels. (b) With lines denoting "half height" boundaries between high and low acoustic impedance regions.

oil field, high acoustic impedance usually corresponds to a high percentage of clay or silt, while low acoustic impedance usually corresponds to a high percentage of sand. The lowest values of acoustic impedance also usually correspond to the existence of oil. By applying the image segmentation method and our procedure for a final segmentation to the acoustic impedance image of Fig. 2, we obtained the segmented image of Fig. 3(a). There are approximately 500 segments that vary considerably in size. A salient feature of this procedure is that the important regions, where oil is present (low $Z$ ) and the adjacent regions, are characterized by strong variations in $Z$ so that they are characterized by a finer segmentation. Most other regions, where oil is not 
present, are characterized by a coarser segmentation. Thus, our final segmentation, despite being the coarsest nontrivial segmentation, is detailed enough at the oil-rich regions and less detailed at the nonproductive regions.

$D$. Segmentation of the logarithmic apparent polarity The final segmentation of the logarithmic apparent polarity is shown in Fig. 1(b). As in Fig. 3(a), each segment is colored, albeit with a different color table, according to the average $P_{L}$ value of that segment. The segmented image reproduces very well the main features of the original image, Fig. 1(a). The average segment values tend to be either large and positive or large and negative so that the segmented image is even more binary in nature than the original image. The oil-bearing regions and layers are spanned by large segments and considerable lateral continuity. This is completely opposite to the acoustic impedance case, where the oil-bearing regions were characterized by the finer segments.

In Fig. 2, black lines denoting the $P_{L}$ segments' (of Fig. 1(b)) boundaries, superimposed on the $Z$ image, demonstrate the following interesting property: every $P_{L}$ segment boundary line passes through minima (maxima) of $Z$; if it reverses its $x$ direction, it passes through maxima (minima) of $Z$. This behavior is completely compatible with the discussion in Sec. A of the relationship between the apparent polarity and the acoustic impedance. We use the $P_{L}$ segment boundaries to segment the acoustic impedance image into alternating layers of high and low $Z$ : for every two adjacent acoustic impedance maximum $Z_{\max }$ and minimum $Z_{\min }$, delineated by two $P_{L}$ segment boundaries, the border between the two corresponding high and low $Z$ regions was set horizontaly at a pixel where $Z_{i} \approx\left(Z_{\max }+Z_{\min }\right) / 2$. Put differently, the borders between adjacent high and low acoustic impedance regions were set at half height between the adjacent acoustic impedance maximum and minimum. These borders, after being consistently completed by their vertical parts, are depicted, overlayed on the $Z$ image, in Fig. 3(b). The results seem surprisingly good.

$E$. Conclusions We have demonstrated a direct method (Sec. C) and an indirect method (Sec. D) for segmenting acoustic impedance images. For the latter method we introduced the logarithmic apparent polarity that is particularly useful for automatic image analysis. Its image [Fig. 1(a)] is composed of easily segmented large connected regions that correspond to either positive or to negative slopes of the acoustic impedance. Consequently, the boundaries of the segmented logarithmic apparent polarity image delineate the maxima and the minima of the acoustic impedance. The location of these delineations was used to infer regions or layers of high and low acoustic impedance. By combining information from the two methods, it appears that the results can be further improved; this is currently under investigation.

Acknowledgements Research was supported in part by the DeepLook Consortium under agreement ERD-97-1506 with Lockheed Martin Energy Research Corporation and in part by the Engineering Research Program of the Office of Basic Energy Sciences and by the Office of Fossil Energy under contract DE-AC05-96OR22464 with Lockheed Martin Energy Research Corporation. I thank British Petroleum Exploration for the seismic data and Marcelo Blatt, David Reister, Fred Aminzadeh, Bradley Carr, and William Doll for discussions and advice.

\section{References}

[1] R. E. Sheriff and L. P. Geldart, Exploration Seismology, Cambridge Univ. Press, 1995.

[2] Handbook of geophysical exploration, Sec. I: Seismic exploration, Vol. 20 Pattern recognition \& image processing, ed. by F. Aminzadeh, Geophysical press, London (1987).

[3] N. Pal \& S. Pal, Patt. Rec. 26, 1277 (1993).

[4] I. Ng, J. Kittler, and J. Illingworth, Signal Proc. 31, 133 (1993).

[5] I. Pitas and C. Kotropoulos, Patt. Rec. 25, 929 (1992).

[6] M. Simaan, Z. Zhang and P. L. Love, page 389 of [2].

[7] F. Aminzadeh and S. Chatterjee, Geoexploration 23, 147 (1984).

[8] M. T. Taner, F. Koehler and R. E. Sheriff, Geophys. 44, 1041 (1979).

[9] M. Blatt, S. Wiseman, and E. Domany, Neural Comp. 9, 1805 (1997); U.S. patent application No. $08 / 725,960$ (pending).

[10] A. Katz (1967). Principals of statistical mechanics, (Freeman, San Francisco, 1967). 